\title{
Análise espacial da pobreza municipal no Ceará, 1991-2000
}

Tulio Chiarini ${ }^{1}$

\begin{abstract}
Resumo: Tem-se falado demasiadamente sobre a distribuição espacial do pobre. Quanto mais desagregado o mapa, mais perfeita é a sua visualização, maior a evidência da heterogeneidade da pobreza e melhor o entendimento da maneira de que ela é formada e como pode ser combatida a partir de políticas públicas localmente específicas. O Ceará apresenta a pobreza distribuída de forma heterogênea por todo o território cearense (medida pela proporção de pobres e pelo índice de pobreza humana municipal), o que é corroborado a partir dos mapas de pobreza apresentados. As hipóteses de que há clusterização da miséria no Ceará e de que alguns municípios cearenses passaram por um processo de difusão de contágios entre 1991 e 2000 são confirmados para os dados fornecidos pelo IPEADATA para o período 19912000. A constatação foi possível através do instrumental econométrico espacial.
\end{abstract}

Palavras-chave: Mapas de pobreza do Ceará; Índice de Pobreza Humana; Análise espacial.

\section{Spatial analysis of municipal poverty in Ceará State, 1991-200o}

\begin{abstract}
There has been a lot of debate on the space distribution of poverty. The more disaggregated the map, the better the evidence of poverty; aggregated national-level poverty data may obscure regional variation and can bias public policies to fight poverty. Ceará State has its poverty (measured by the headcount index and by the human poverty index) distributed in a heterogeneous way throughout its territory, which is corroborated by the maps of poverty displayed in this paper. The hypotheses that there is a cluster of misery in Ceara State and that some towns went through a contagious diffusion between 1991 and 2000 are confirmed when using the data supplied by IPEADATA for the years 1991
\end{abstract}

1 Economista pela Faculdade de Ciências Econômicas da UFMG, mestrando em economia pelo Programa de Pós-graduação em Economia da UFRGS, bolsista Capes. E-mail: tuliochiarini@yahoo.com.br. 
and 20oo. The corroboration was possible thank to the use of spatial econometrics tools.

Key-words: Ceará poverty maps; Human poverty index; spatial analysis.

JEL: I32, I39, Roo

\section{Introdução}

A importância do espaço em assuntos econômicos ganhou novo fôlego com as investidas de Paul Krugman e sua troop tentando mostrar, sobretudo aos economistas do meanstream, a importância da geografia. A ajuda de mapas logo facilitou a identificação espacial de variáveis sócio-econômicas. Com o auxílio metodológico tanto da econometria tradicional quanto da econometria espacial - novo ramo que se fez necessário, foi possível encontrar padrões de influência do espaço.

O estudo sobre bem-estar social e pobreza não ficou a mercê desse novo rumo e apropriou-se do uso de mapas para localizar o pobre e verificar se existe um certo padrão de distribuição da pobreza ou se ela se distribui aleatoriamente no espaço. A utilização desses mapas tem sido importante não somente para prover figuras elucidativas mostrando onde os pobres estão, mas por servir como instrumento capaz de capturar a heterogeneidade dentro de uma determinada região, já que indicadores agregados em nível nacional podem dar a (falsa) impressão de que as condições internas desse país estão uniformemente distribuídas e freqüentemente podem esconder variações consideráveis em nível subnacional.

A escolha metodológica da mapeação da pobreza é complexa e depende de um número de variáveis e considerações lógicas e legítimas que podem dificultar sua operacionalização e sua efetiva construção ${ }^{2}$. Contudo, a mapeação do pobre tem sido muito relevante em ajudar a entender tanto a distribuição espacial da pobreza quanto a distribuição

2 Dentre as objeções encontradas, a visão filosófica do pesquisador é uma das mais significativas, pois pode dar nova configuração ao mesmo a partir da sua definição (subjetiva) de pobreza. De acordo com Davis (2002; 2003), o pesquisador tem uma gama de crenças que pode afetar sua escolha metodológica devido à influência de sua 'doutrina' profissional e/ou devido às características da instituição onde trabalha. Além disso, o limite da oferta de dados, a oferta de dados fidedignos e o acesso a estes são restrições que devem ser levadas em conta ao se construir o mapa. Uma base de dados contestáveis pode, da mesma forma que a visão filosófica, afetar a conformação do mapa de pobreza. A capacidade analítica dos pesquisadores e os custos envolvidos no estudo também não podem ser negligenciados. A sofisticação econométrica das estimações espaciais requer, tanto dos pesquisadores quanto do público, conhecimentos estatísticos e econométricos elevados para realizar e interpretar os resultados, como sugere Davis (2002; 2003). Dessa forma, quanto mais sofisticada a análise e quanto mais dados a serem coletados, mais alto é o custo da mapeamento. O custo, como evidencia Davis (2002), ainda inclui o tempo gasto em obter e entender os dados e analisá-los. Por essas razões a elaboração de um mapa de pobreza 'ideal' é abstrusa. No entanto, essas ressalvas são contrabalanceadas pelas vantagens que os mapas de pobreza proporcionam. 
de ativos que são fundamentais para minimizá-la e aliviá-la, servindo também como instrumento capaz de capturar a heterogeneidade dentro de um país. A melhora no alvo de intervenções e distribuição de recursos também é outra vantagem proporcionada pelo uso de mapas, contribuindo como instrumento de mira para políticas sociais, emergenciais e programas pró-pobres e, ainda melhorando o alvo de gastos públicos ao identificar onde a população mais necessitada está localizada, seja regional ou localmente. Tais mapas podem ainda fornecer ferramentas analíticas para discernir por que a pobreza persiste em certas áreas. Finalmente, a produção e distribuição de mapas de pobreza podem resultar em aumento da transparência das decisões públicas ao aumentar a consciência sobre a pobreza, fomentar debates e encorajar o engajamento da sociedade civil em tais decições. (Henninger 1998; Hentschel et al. 1998; Deichmann 1999; Henninger \& Snel 2002; Petrucci et al. 2003; Gauci 2005).

No Brasil, o tema ainda é incipiente. Não obstante, já se pode dizer que é um dos rumos atuais que pesquisas sobre pobreza e desigualdade têm tomado. Recentemente, Romero (2006), por exemplo, fez uma análise espacial da pobreza dos municípios do estado de Minas Gerais. Câmara et al. (2004) apresentam uma metodologia para mapear a exclusão e inclusão social em áreas urbanas de países em desenvolvimento, usando técnicas de análise espacial para identificar certos padrões. Oliveira (2006) utiliza-se do método econométrico espacial com dados em painel e estima as elasticidades e concentração de renda com relação à pobreza dos municípios nordestinos. Além disso, Oliveira (2006) calcula as contribuições do crescimento econômico e da redução da desigualdade de renda na redução da pobreza nesses municípios. Cunha (2006) avalia uma das metodologias de mapeação da pobreza e a aplica para retratar a pobreza no Rio Grande do Norte, a partir do small area estimation method. Por sua vez, Liberato (2004) apresenta mapas da incidência da pobreza e da indigência tanto para Belo Horizonte quanto para a região metropolitana mineira, enquanto que Borges (2004) elabora o mapa da pobreza urbana de São José dos Campos, que também foi o objeto de análise de Genovez (2002), cujo foco, contudo, está em utilizar técnicas de análise espacial para estudar a exclusão social nesta cidade. Já no Rio Grande do Sul, foi elaborado o mapa da inclusão e exclusão social de Porto Alegre (Porto Alegre 2004).

Esse artigo tem como objetivo contribuir para essa 'nova corrente de pesquisa' mapeando a pobreza em dois pontos no tempo, 1991 e 2000, no Ceará. A importância de tal análise está em permitir encontrar clusters de pobreza e riqueza para que políticas públicas específicas possam tratar a heterogeneidade do espaço cearense de forma adequada, a partir da utilização de dois índices de pobreza: headcount index e o 
índice de pobreza humano municipal. A hipótese básica a ser testada é, portanto, a de que existe algum tipo de dependência espacial da pobreza, não importando o tipo de indicador utilizado, para ambos os anos. Outro objetivo desse artigo é fazer uma análise do processo da dinâmica da difusão espacial entre o período analisado, verificando quais os municípios cearenses passaram por um processo de difusão contagiosa, seja ela por expansão ou relocalização. Para tanto, esse artigo foi dividido em algumas seções além dessa introdução. A seção seguinte apresenta as formas utilizadas para mensurar a pobreza e logo em seguida são mostradas algumas informações acerca da pobreza espacial no Ceará. A metodologia de análise que será empregada é apresentada posteriormente e tem-se a análise para o Ceará e os resultados encontrados. Finda-se esse artigo com as considerações finais, retomando os principais pontos abordados no decorrer deste.

\section{Definição de pobreza}

Nota-se que a mensuração do bem-estar e da pobreza não é trivial, não há um consenso de como mensurá-lo, nem de quais atributos axiomáticas são de fato relevantes para um indicador 'ideal' e ainda há diversas questões metodológicas relacionadas à sua definição e à forma de sua mensuração. A escolha de uma certa definição é feita com base em argumentos pragmáticos, de acordo com acesso aos dados, com decisões políticas e até mesmo de acordo com argumentos históricos, como ressalvam Hagenaars e De Vos (1988). Por isso não existe uma definição única de pobreza, tudo dependerá da maneira da qual se define o bemestar.

Como sugere Henninger (1998), a maioria dos indicadores de bem-estar e pobreza pode ser agrupada em três grandes dimensões: econômica, social e enabling environment. A dimensão econômica está calcada em duas variáveis chaves para se mensurar o bem-estar e a partir daí definir o que é pobre. São elas renda e consumo. O último reflete o padrão de vida dos indivíduos, ou seja, o total que é gasto no consumo de bens e serviços, o que reflete a magnitude da utilidade derivada deste. A renda, por sua vez, pode ser utilizada como proxy do consumo. Os gastos de consumo usados como uma medida do padrão de vida tem sua origem na teoria do bem-estar social (welfare theory). O total que é gasto no consumo de tais bens reflete, portanto, a utilidade proveniente desse consumo. A escolha da cesta de consumo por domicílio baseia-se nas preferências individuais. Dessa forma, como lembram Ravallion (1992) e Henninger (1998), assume-se que cada domicílio está maximizando a utilidade individual de cada morador e, quanto maior o consumo de bens e serviços, maior será a utilidade. Uma alternativa de mensuração 
do padrão de vida como indicador direto do bem-estar é a riqueza total dos indivíduos, como demonstram Ravallion (1992) e Mckinley (1997). Entretanto, dados sobre riqueza são raros e/ou não confiáveis ${ }^{3}$.

Todas essas três formas de mensuração do padrão de vida estão ancoradas no uso de métrica monetária para estimar o fluxo de renda, o valor dos bens e serviços ou da magnitude total do patrimônio. Tudo se resume em unidades monetárias comuns e os preços passam a desempenhar a ponderação para cada item (McKinley 1997). A vantagem desse método é que medidas monetárias são facilmente comparáveis e solucionam o problema de se assumir pesos arbitrários para bens e serviços e dessa forma permite produzir índices de pobreza mais adequados (Henninger 1998). Não obstante, a utilização da métrica monetária para mensuração do bem-estar e da pobreza não é inquestionável.

Primeiramente, como salienta McKinley (1997:2), a moeda é apenas uma maneira aproximada de se medir o valor de bens e serviços:

Money's purpose is to facilitate exchange; it has no value in itself. Goods and services may be useful to people, but their monetary value is ascribed to them as a proxy for their use value. (...) The second problem is that goods and services are also not valuable in themselves. They are a means to human well-being: food is an input, for example, into people's nutrition. Thus, money turns out to be an indirect means to human well-being: it is useful only for purchasing the direct means to human well-being, such as food, clothing and shelter. Also part of the problem is that money does not cover some vital inputs into people's well-being, such as public goods, community resources, social relations, culture and the natural environment.

Além disso, a pobreza não é um fenômeno homogêneo que pode ser mensurado adequadamente somente a partir de uma única dimensão, mesmo sabendo da importância do dinheiro para

3 As medidas de dimensão social são: nutrição, saneamento e água potável, energia, educação, saúde e planejamento familiar. A importância dos indicadores de dimensão social, como discorre Henninger (1998), é que fornecem medidas de capacitação humana. Indicadores como acesso a saneamento básico e a água potável, por exemplo, são indicativos de condições socioeconômicas (in) satisfatórias, além de (in) suficiente cobertura e qualidade da utilização de procedimentos básicos de atenção à saúde. Dentre os componentes da dimensão enabling eviornment têm-se o acesso ao capital geográfico para a produção, a vulnerabilidade e as áreas periféricas. O capital geográfico para a produção é a proporção da população com acesso a capital natural (como terra, água, florestas, etc.), acesso ao capital físico (como estradas, informação, transporte, crédito, tecnologia, etc) e acesso a capital social (como grupos sociais, rede social, etc.). Já a vulnerabilidade, de acordo com Henninger (1998), pode ser definida como a susceptibilidade de um indivíduo de sofrer choques e flutuações externos e é agrupada em algumas categorias: risco ambiental (secas, enchentes, deslizamentos), risco de mercado (flutuação de preços, variação de salários, desemprego), risco político (mudanças em subsídios, transferências de renda), risco social (redução de entitulamentos) e risco de saúde (exposição a doenças). Finalmente, as áreas periféricas são aquelas áreas 'esquecidas' como afirma Henninger (idem), que são caracterizadas pelo isolamento e altos índices de pobreza. 
minimizá-la. O bem-estar, como já mencionado, ocorre em dimensões múltiplas e, conseqüentemente, a falta deste, ou seja, a pobreza deve relatar a falta de todas essas dimensões, tais quais saúde, nutrição, educação, etc. Finalmente, como relembra Henninger (1998), o uso de métricas monetárias pode ainda gerar dificuldades em capturar o verdadeiro valor monetário de bens 'não-comercializáveis' e 'nãoprecificáveis', como, por exemplo, bens de consumo de subsistência, existência de capital social e capital natural, dentre outros. Mesmo sabendo de tais fragilidades e das inúmeras questões que possam ser fomentadas, a pobreza nesse artigo é tratada como insuficiência de renda, assim o índice de pobreza aqui utilizado é a proporção dos pobres, FGT(o), sendo que nesse caso é o percentual de pessoas com renda domiciliar per capita inferior a $\mathrm{R} \$ 75,50$, equivalente a $1 / 2$ salário mínimo vigente em agosto de 2000. Na literatura esse índice é conhecido como headcount index e pode ser obtido a partir do índice Foster-GreerThorbecke e é derivado da seguinte equação:

$$
P_{\alpha}=\frac{1}{N} \sum_{i=1}^{M}\left[\frac{\left(z-y_{i}\right)}{z}\right]^{\alpha}
$$

onde $z$ é a linha de pobreza, $y_{i}$ é a renda (ou consumo) do indivíduo $i$ de um domicílio pobre, $N$ é o número de pessoas da população e $M$ o número de pessoas em domicílios pobres. Assim, quando $\alpha=0$ tem-se o headcount index. Para explicação sobre esse e demais índices de pobreza, ver Hagenaars (1987) e Ravallion (1992).

Outra forma de que se trata a pobreza nesse artigo é a partir da combinação de medidas de bem-estar social em um único índice. O Human Poverty Index, apresentado no Relatório de Desenvolvimento Humano da ONU de 1997, inclui três variáveis: porcentagem de pessoas que se espera morrer antes de quarenta anos de idade, porcentagem de adultos analfabetos e uma medida de padrão de vida, definido pela proporção de pessoas com acesso a serviços de saúde, proporção de pessoas com acesso a água potável e proporção de crianças desnutridas até cinco anos de idade.

Antes de se calcular o índice de pobreza humana é preciso criar cada uma das dimensões que servirão para mensurá-lo (HDR 2004). O índice é calculado da seguinte maneira:

$$
P(\alpha)=\left(\frac{w_{1} P_{1}^{\alpha}+w_{2} P_{2}^{\alpha}+w_{3} P_{3}^{\alpha}}{w_{1}+w_{2}+w_{3}}\right)^{1 / \alpha}
$$


onde $P(\alpha)$ é o índice de pobreza ponderado a ser calculado de ordem $\alpha$ de $P_{1}, P_{2}$ e $P_{3}$, sendo que $w_{i}>0$ é a ponderação de $P_{i}(\geq 0)$, onde $\mathrm{i}=1,2,3$. Dessa forma, $P_{1}$ é a porcentagem de pessoas que se espera morrer antes de quarenta anos de idade, $P_{2}$ é porcentagem de adultos analfabetos e, finalmente, $P_{3}$ é a porcentagem de indivíduos que possuem certo padrão de vida $-P_{3}=\frac{P_{3,1}+P_{3,2}+P_{3,3}}{3}$, onde $P_{3,1}$ é proporção de pessoas com acesso a serviços de saúde, $P_{3,2}$ é proporção de pessoas com acesso a água potável e $P_{3,3}$ é proporção de crianças desnutridas até cinco anos de idade.

Discutiu-se se o Human Poverty Index poderia ser utilizado para todo e qualquer tipo de país, independente do seu estágio de desenvolvimento. O Relatório de Desenvolvimento Humano da ONU de 2004 sugere calcular dois tipos de índices de pobreza humana, um para países em via de desenvolvimento e outro para países desenvolvidos, o HPI-1 e o HPI2, respectivamente ${ }^{4}$. O HPI-1 é calculado da mesma forma que o HPI já apresentado, porém o HPI-2 além de mensurar as privações do HPI-1 também mensura exclusão social. Dessa forma o HPI-2 reflete a privação em quatro dimensões distintas: vida longa e saudável, conhecimento, padrão de vida descente e exclusão social.

Uma vida longa e saudável tem como proxy a probabilidade ao nascimento de não sobreviver até os sessenta anos de idade. Por sua vez, o conhecimento é mensurado a partir da porcentagem de adultos (entre dezesseis e 65 anos de idade) analfabetos funcionais. Já o padrão de vida recente é calculado a partir da porcentagem de pessoas que vivem abaixo da linha de pobreza. Finalmente, a exclusão social é captada a partir da taxa de desemprego de longo prazo (doze meses ou mais). A formulação do HPI-2 é praticamente igual ao do HPI-1, sendo que $P_{i}(\geq 0)$ e $\mathrm{i}=1,2,3,4$.

Para uma análise municipal, a Fundação João Pinheiro, de Minas Gerais, fez uma alteração metodológica do IPH para se adaptar alguns indicadores, disponíveis em nível municipal, para seu cálculo. Dessa forma, o índice de pobreza humano municipal (IPH-M) é calculado a partir das seguintes estatísticas: probabilidade de sobreviver até os 40 anos de idade, porcentagem de pessoas com 25 anos ou mais de idade

\footnotetext{
4 Embora se utiliza as mesmas dimensões para a criação do IPH-1 e IPH-2, os indicadores de cada dimensão são diversos. Por exemplo, a dimensão 'conhecimento', para países em desenvolvimento, é medida pela taxa de analfabetismo adulto, o que não seria um bom indicador para países desenvolvidos, já que a taxa de analfabetismo de adultos nesses países não seria um indicador fidedigno dessa dimensão. Por isso se utiliza a porcentagem de adultos com analfabetismo funcional. A mesma lógica se dá com as demais dimensões que compõe os índices.
} 
analfabetas, e uma ponderação como proxy para um padrão de vida decente [ que é a porcentagem de pessoas que vivem em domicílios sem água encanada e a porcentagem de crianças até 5 anos de idade falecidas (proxy de desnutrição), sendo esse índice adaptado outra forma de mensuração da pobreza utilizada nesse artigo].

\section{Analisando o Ceará}

Em 2000, houve uma redução da proporção dos pobres em todas as regiões e em todos os estados nordestinos, vis-à-vis a proporção de pobres em 1991. Mesmo com essa redução, a proporção de pobres no Nordeste continua sendo a maior em todo o país, chegando ao patamar de $56 \%$ da população considerada pobre (Ver Tabela 1). Contudo, esse valor é apenas uma média da proporção de pobres nos estados nordestinos, escondendo, portanto, a verdadeira pobreza nesses estados. No mesmo ano, tomando-se por exemplo o Ceará, aproximadamente $57 \%$ da população é formada por pobres. Certamente que analisando esse estado individualmente pode-se ainda perceber que o percentual de pobres não se distribui de forma homogênea.

TABELA 1. PROPORÇÃO DE POBRES NAS REGIÕES BRASILEIRAS E NOS ESTADOS DO NORDESTE, 1991-2000

\begin{tabular}{lcc}
\hline & 1991 & 2000 \\
\hline Região Centro-oeste & 32.67 & 25.51 \\
Região Norte & 52.76 & 49.60 \\
Região Nordeste & 67.12 & 56.93 \\
Região Sul & 30.79 & 20.53 \\
Região Sudeste & 24.24 & 19.78 \\
Alagoas & 67.18 & 62.24 \\
Bahia & 67.04 & 55.32 \\
Ceará & 68.23 & 57.00 \\
Maranhão & 75.07 & 66.81 \\
Paraíba & 69.04 & 55.26 \\
Pernambuco & 60.04 & 51.31 \\
Piauí & 74.46 & 61.81 \\
Rio Grande do Norte & 61.71 & 50.63 \\
Sergipe & 61.06 & 53.99 \\
\hline
\end{tabular}

FONTE: IPEADATA, 2007. NOTA: Percentual de pessoas com renda domiciliar per capita inferior a $\mathrm{R} \$ 75,50$, equivalentes a $1 / 2$ do salário mínimo vigente em agosto de 2000. 
Assim, avaliando espacialmente a pobreza no Ceará em 1991 e 2000, conforme apresentado na Figura 1, pode-se perceber que há uma expressiva variabilidade da mesmo em termos municipais. Pode-se se compreender que a proporção de pobres e o IPH-M em 1991 sofreram nova configuração em 2000 , corroborando a hipótese de diminuição da pobreza no período analisado.

\section{FIGURA 1. POBREZA POR MUNICÍPIO CEARENSE, PROPORÇÃO DE} POBRES, 1991-2000
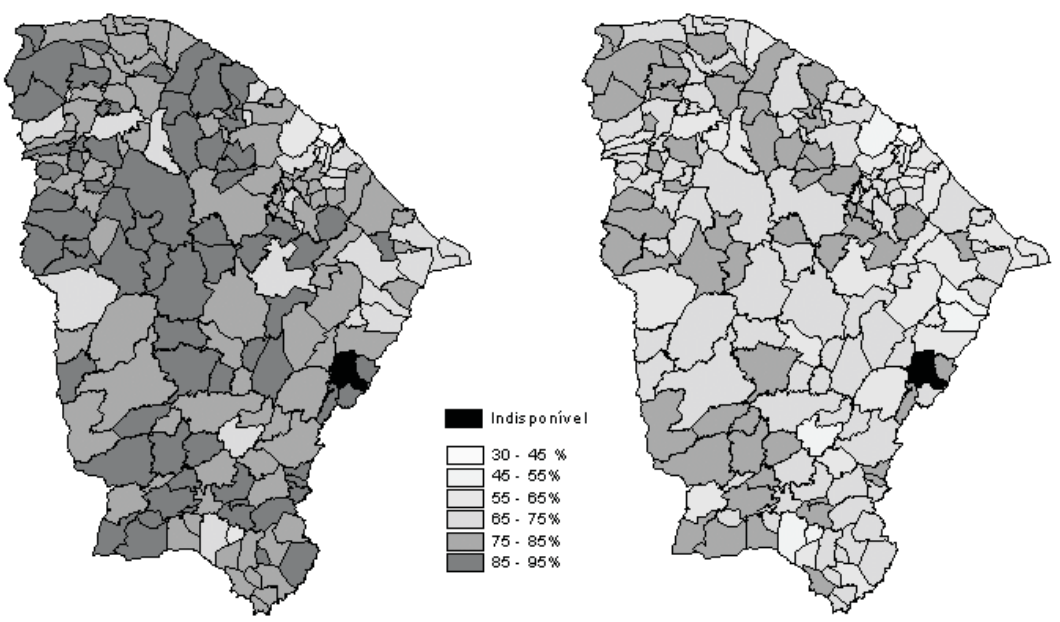

FONTE: Elaboração do autor a partir dos dados do IPEADATA e a partir dos mapas georeferenciados disponíveis pelo IBGE.

NOTA: Município Jaguaribe indisponível.

Os mapas da pobreza do Ceará são apresentados nas Figuras 1 e 2, contudo, sua mapeação é mais do que um exercício cartográfico. Por isso, alguns pontos chaves são levantados: existe um padrão de dependência espacial entre os municípios cearenses levando-se em conta a proporção dos pobres e o IPH-M? Ou seja, há municípios pobres cercados por municípios também pobres, isto é, 'clusterização da miséria'? Há municípios ricos cercados por municípios também ricos, ou seja, 'clusterização da riqueza'? Igualmente, pode-se esperar dissimilaridade espacial como municípios ricos cercados por municípios pobres e municípios pobres cercados pelos municípios ricos? Como essa configuração se deu entre os anos analisados? A hipótese aqui proposta é a de que 
existe dependência espacial da pobreza no Ceará, para ambos os indicadores de pobreza e para ambos os anos, o que será testado na seção 5 .

Para tal análise é necessário um instrumental que vem sendo utilizado de forma crescente em análises econômicas localizacionais: a econometria espacial. Por isso, a análise econométrica espacial é apresentada de forma breve na seção seguinte.

FIGURA 2. POBREZA POR MUNICÍPIO CEARENSE, IPH-M, 1991-2000
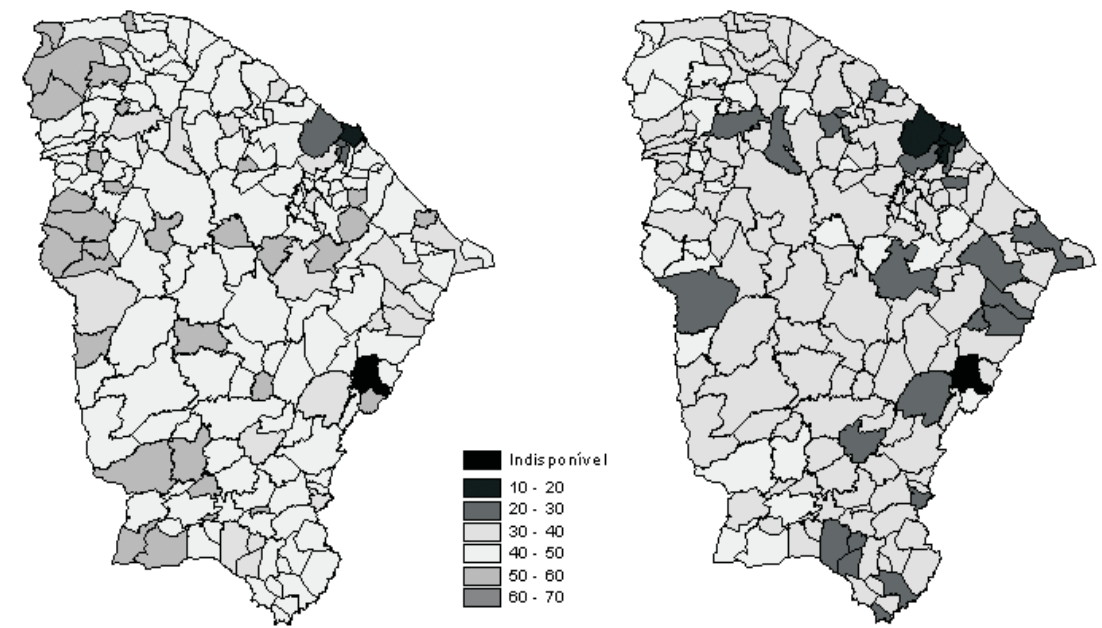

FONTE: Elaboração do autor a partir dos dados do IPEADATA e a partir dos mapas georeferenciados disponíveis pelo IBGE.

NOTA: Município Jaguaribe indisponível.

\section{Metodologia}

O instrumental econométrico espacial é capaz de descrever e visualizar as distribuições geográficas da pobreza e das demais variáveis, identificando localizações atípicas, descobrindo formas de associação (ou seja, se a pobreza nos municípios cearenses se distribui de forma aleatória ou se existe evidência de algum tipo de dependência espacial entre as medidas) que, por sua vez, podem ser de caráter global ou local e sugerindo estruturas no espaço geográfico. De encontro com Anselin (1988, 1999a, 1999b, 2000), Anselin e Rey (1997), LeSage (1998) e Chasco (2003), a econometria espacial, termo cunhado por Jean Paelinck, na década dos 1970, lida com a dependência e a heterogeneidade espacial e, por essa razão, a econometria convencio- 
nal se aplicada a assuntos cuja localização importa não é a mais adequada, já que não trata de tais efeitos.

Mas qual o motivo de se utilizar a econometria espacial? A razão de se aplicar técnicas econométricas espaciais deve-se a certas peculiaridades dos dados de modelos que possuem caráter espaço-geográfico (Chasco 2003). Como exemplo dessas particularidades, tem-se sua natureza georreferenciada, a multidirecionalidade de suas relações e a multidimensionalidade desses dados. Essas características peculiares levam aos chamados efeitos espaciais de dependência e heterogeneidade (ou seja, autocorrelação espacial e estrutura espacial, respectivamente).

Nesta seção, define-se a econometria espacial, seguindo a demarcação dada por Anselin (1988, 1999a), LeSage (1998) e Chasco (2003), como um disciplina proveniente da econometria tradicional que precisa de tratamento adequado de dados geográficos em modelos de regressão onde o espaço importa. Essa seção é subdivida, por sua vez, em subseções: a primeira apresenta os efeitos de dependência espacial e a segunda parte apresenta brevemente a heterogeneidade espacial. Vale lembrar que ambos são conceitos próprios de dados espacialmente localizados.

\title{
3.1 Dependência espacial (autocorrelação espacial)
}

De acordo com Anselin (1988), a dependência espacial se manifesta pela falta de independência que geralmente está presente entre as observações de dados em cross-section. Segundo Chasco (2003:9):

\begin{abstract}
a primera vista, la dependencia espacial puede parecer similar a la más conocida dependencia presente en los tests econométricos de correlación de series, en los modelos de distribución de retardos y en otros análisis de series temporales. Sin embargo, esta similitud sólo es real en parte, debido a la naturaleza multidireccional de la dependencia en el espacio que, frente a la clara situación unidireccional del tiempo, hace necesario el uso de una estructura metodológica diferente.
\end{abstract}

Segundo LeSage (1998), a presença de dependência espacial significa que uma observação associada a uma localização, por exemplo, $i$, depende de observações nas localizações $j$, sendo que $i \neq j$. Formalmente: $Y_{i}=f\left(x_{j}\right) i=1, \ldots, n i \neq j$

Qual a razão de se esperar que a amostra de dados observados em um ponto no espaço seja dependente de valores observados em outras localizações? Anselin (1988) afirma que a dependência espacial pode 
ser causada por uma infinidade de problemas de mensuração ${ }^{5}$, como a delineação arbitrária da espacialidade das observações. Como re-afirma LeSage (1998), isso ocorre se, por exemplo, as fronteiras administrativas da informação coletada não refletirem exatamente a natureza do processo gerador da amostragem de dados. Outra razão da existência da dependência espacial, conforme LeSage (1998), é que a dimensão espacial da atividade econômica é um importante aspecto na modelagem e não deve ser negligenciado. Problemas de agregação espacial e a presença de externalidades espaciais e efeitos transbordamento são, da mesma forma, outros problemas que levam à dependência espacial, em concordância com Anselin (1988).

Daí depara-se com uma das questões cruciais em econometria espacial: como expressar formalmente a dependência espacial e como incorporála em modelos. Moran e Geary (apud ANSELIN 1988) basearam a dependência espacial através da noção de contigüidade ${ }^{6}$ binária entre as unidades espaciais, ou seja, a estrutura dos vizinhos era expressa a partir dos valores binários, o e 1. Dessa forma, se duas unidades espaciais têm uma fronteira comum, então tais unidades são consideradas contíguas e recebem o valor um. Contrariamente, as unidades não vizinhas recebem o valor zero para classificá-las. Isso implica afirmar que, conforme LeSage (1998), observações que estão mais próximas uma das outras devem refletir um maior grau de dependência espacial do que as mais distantes, conseqüentemente, o poder da dependência espacial entre as observações deve declinar com a distância entre elas. "Regarding spatial dependence, neighboring units should exhibit a higher degree of spatial dependence than units located far apart" (Lesage 1998:8).

\footnotetext{
5 Por um bom tempo, a dependência espacial foi ignorada por diversos econometristas que consideravam as unidades de corte-transversais como sendo mutuamente independentes. Esse período, em que se desprezava a econometria espacial, é chamado por Paelinck (2005), de idade 'das trevas da econometria' (dark ages), já que tendo como unidades de corte-transversais regiões geográficas arbitrariamente definidas por certas fronteiras, a aceitação da suposição da independência é questionável. Claro que não se pode desqualificar metodologias reconhecidas cientificamente, classificadas como clássicas. A metodologia espacial é um aprimoramento muito bom, um avanço metodológico, mas que não inviabiliza nem desqualifica metodologias "clássicas". Grosso modo, a dependência espacial é similar à dependência temporal das séries de tempo, todavia, a última apresenta natureza direcional no tempo (ou seja, o passado explica o presente) enquanto a primeira possui natureza multidirecional (ou seja, uma região pode estar afetada não somente por uma outra região contígua a ela, mas por muitas outras que a rodeiam) (Anselin 1988; Lesage 1998). 6 A palavra 'contigüidade', do inglês contiguity, tem origem na palavra 'contíguo', cujo significado, de acordo com o dicionário Houaiss da língua portuguesa, é "que está adjacente ou próximo; vizinho". A contigüidade pode ser formalizada de diversas formas, especialmente quando a unidade de referência espacial refere-se a uma grade ou uma coleção de pontos irregulares, como ressalvam Anselin (1988) e LeSage (1998). Em muitos casos, quando há espaços regulares ou irregulares entre as localidades, o significado de contigüidade pode se derivar do caminho mais curto formado pelas localidades.
} 
Para incluir uma mensuração geral sobre a interação potencial entre duas unidades espaciais, deve-se ampliar o conceito de contigüidade binária, mediante a conceituação de matriz de ponderação espacial, ou matriz de ponderação Cliff-Ord, como manifesta Anselin (1988). A determinação dos elementos dessa matriz é controversa e não consensual; com propriedade Anselin (1988, p. 21) afirma que "there is no agreement as to which type of weight matrix should be used in spatial econometric analysis". Prossegue logo adiante (1988:26), "the variety of spatial weight matrices and levels of spatial aggregation create specific, methodological problems for spatial econometrics"”.

\subsubsection{Moran Global e Local}

A perspectiva global do fenômeno de autocorrelação espacial objetiva contrastar a presença de tendências ou estruturas espaciais gerais na distribuição de uma variável sobre o espaço (Chasco 2003; Vaya \& Serrano 2000). Ou seja, o ponto de vista global trata de afrontar a hipótese de uma variávelque se encontra distribuída de forma totalmente aleatória no espaço ou se, ao contrário, existe qualquer tipo de associação estatisticamente significativa entre os valores similares ou distintos entre as regiões contíguas.

Pode-se verificar, portanto, se os dados estão ou não correlacionados espacialmente a partir de alguns testes, como o I-Moran, contudo esse teste não oferece a estrutura regional da autocorrelação. O gráfico e a estatística I-Moran Global são os instrumentos mais amplamente utilizados (ANSELIN 1988). A estatística I-Moran Global indica a 'força' da similaridade ou dissimilaridade espacial das regiões vizinhas, sendo que uma estatística I-Moran Global significamente positiva indica regiões vizinhas similares enquanto que uma estatística I-Moran Global significamente negativa indica dissimilaridade entre as regiões contíguas. Se $x_{1}, x_{2}, \ldots x_{N}$ forem as observações de $N$ locais, o I-Moran Global para estas $N$ observações é dado por:

$$
I=\frac{N}{w_{0}} \frac{\sum_{i=1}^{N} \sum_{j=1, i \neq j}^{N} w_{i j}\left(x_{i}-\bar{x}\right)\left(x_{j}-\bar{x}\right)}{\sum_{i=1}^{n}\left(x_{i}-\bar{x}\right)^{2}}
$$

\footnotetext{
7 Mesmo não sendo consensual, usualmente, a matriz de ponderação é formada por uma combinação da distância e contigüidade, sendo que a distância pode ser expressa, conforme Anselin (1988), pelo tempo de viagem, distância geral ou derivada de uma análise de escala multidimensional, podendo ser utilizada tanto a distância euclidiana quanto o arco da distância, por exemplo. Assim, por exemplo, quando as unidades pertencem a uma rede quadricular, a contigüidade não é única.
} 
onde, $w_{i j}$ são os elementos da matriz de contigüidade binária normalizada $(W)$ representando a proximidade de diferentes localizações, ou seja, se $i$ e $j$ são locais vizinhos $(i \neq j), w_{i j}$ terá o valor unitário, como já discutido na seção anterior; caso contrário terá o valor nulo. Finalmente, $x_{i}$ e $x_{j}$ são os valores da variável analisada e

$w_{0}=\sum_{i=1}^{N} \sum_{j=1} w(i . j)$.

Quando o I-Moran Global for próximo de +1, isso quer dizer que há autocorrelação positiva (clustering). Em análise de pobreza, isso implica que regiões pobres (não-pobres) tendem a estar localizadas na vizinhança de pobres (não-pobres). Se a estatística I for próxima de $\mathbf{- 1}$, tem-se o oposto, autocorrelação negativa (outlier espacial): regiões pobres (não-pobres) estão cercadas de regiões não-pobres (pobres). E, finalmente, se o valor do I-Moran for igual à zero, tem-se que não há autocorrelação espacial.

Através do correspondente gráfico de dispersão do I-Moran é possível decompor o tipo de associação espacial em quatro categorias, o denominado Scatterplot de Moran que representa no eixo das abscissas as observações da variável $x$ normalizada e o eixo das ordenadas o lag espacial dessa variável também normalizada. Desse modo, os quatro quadrantes reproduzem diferentes tipos de dependência espacial. Dois tipos de similaridades espaciais existem: regiões pobres cercadas por regiões pobres, ou seja, clusterização da pobreza do tipo alto-alto (primeiro quadrante) e regiões não-pobres cercadas por regiões nãopobres, ou seja, clusterização da pobreza do tipo baixo-baixo (terceiro quadrante). Igualmente, dois tipos de dissimilaridade espacial existem: regiões não-pobres cercadas por regiões pobres, ou seja, clusterização da pobreza do tipo baixo-alto (segundo quadrante) e regiões pobres cercadas por regiões não-pobres, ou seja, clusterização da pobreza do tipo alto-baixo (quarto quadrante).

Outra forma de se verificar se unidades ao redor de outra possuem valores semelhantes é através de um indicador local de associação espacial (local indicator of spatial association - LISA). Através deste procedimento, desenvolvido por Luc Anselin, é possível avaliar a hipótese de aleatoriedade espacial comparando os valores do indicador de cada região com os indicadores das regiões vizinhas. Segundo Anselin et al. (2000, p. 233-234), "LISA provide a measure of the extent to which the arrangement of values around a specific location deviates from spatial randomness." Um indicador local de associação espacial é a estatística I-Moran Local $\left(I_{i}\right)$, que mensura a autocorrelação espacial de uma localização específica com os seus vizinhos. Da mesma forma que o I- 
Moran Global, o I-Moran Local significamente positivo indica que os valores do local em questão e seus vizinhos são similares, ou seja, há autocorrelação positiva (há padrões de similaridade espacial). Já o IMoran Local significantemente negativo indica que o valor da localização em análise é desigual em relação a seus vizinhos, ou seja, há padrões de dissimilaridade espacial. O I-Moran Local pode ser calculado da seguinte forma a seguir, para uma localização $i$, e quando os valores de $I_{i}$ são diferentes de zero, isso indica que a unidade $i$ está espacialmente associada aos seus vizinhos.

$$
\begin{aligned}
I_{i} & =\frac{x_{i}-\bar{x}}{S_{i}^{2}} \sum_{j=1}^{N} w_{i j}\left(x_{i}-\bar{x}\right) \\
\text { onde } \quad S_{i}^{2} & =\frac{\sum_{j=1, j \neq i}^{N} x_{j}^{2}}{N-1}-\bar{x}^{2}
\end{aligned}
$$

\section{Análise espacial da pobreza no Ceará}

Em primeiro lugar, avalia-se se existe evidência estatística de dependência espacial da pobreza a nível municipal, a partir da estatística IMoran Global. Toma-se como matriz de contigüidade a queen contiguity $^{8}$, tendo com hipótese a contigüidade de primeira e segundaordem $^{9}$. A amostra dos dados constitui-se de

\footnotetext{
8 Existem diversas maneiras de se estabelecer uma fronteira comum entre a região em análise e as que a rodeiam. Por exemplo, quando se defini $w_{i j}=1$ para as localidades que têm a mesma borda imediatamente à direita ou à esquerda da região em questão, tem-se a contigüidade linear (ou linear contiguity). Já, seguindo a lógica do jogo de xadrez, quando se define $w_{i j}=1$ para as regiões que têm um lado comum com a região de interesse tem-se a contigüidade de torre (ou rook contiguity). Da mesma forma, também seguindo a lógica do jogo de xadrez, define-se $w_{i j}=1$ para as regiões que possuem o mesmo vértice com a região de interesse, tem-se contigüidade do bispo (bishop contiguity) e a contigüidade de rainha (queen contiguity) é definida para as regiões que compartilham o mesmo lado ou o mesmo vértice com a região de interesse, definindo-se $w_{i j=1}$ (Lesage 1998; Anselin 2002).

9 A idéia de contigüidade espacial pode ser generalizada para vizinhos de maior ordem (ou seja, vizinhos dos vizinhos). Dessa forma, é possível considerar várias ordens de contigüidade, definindo a ordem de contigüidade k-ésima para quando as unidades espaciais são contíguas de primeira ordem a uma unidade espacial de ordem (k-1)-ésima e não contíguas de ordem inferior (Anselin, 1988). Seguindo critério análogo ao adotado para a construção da matriz de contigüidade de primeira ordem, é possível construir as matrizes $W^{(2)}, W^{(3)}, \ldots, W^{(n)}$. Por exemplo, em um sistema de redes quadricular, a ordem da contigüidade pode ser vista através de uma série de novas unidades ao redor de um único centro de interesse.
} 
183 municípios cearenses ${ }^{10}$ de um total de 184 , sendo que Jaguaribe não apresentou uma ou outra variável para a análise e por isso foi desconsiderado. Obtivemos as seguintes estatísticas I-Moran apresentadas na Tabela 2.

A partir da Tabela 2 e das Figuras 1 e 2, do Anexo A, pode-se perceber que há indícios de autocorrelação espacial positiva para a proporção de pobres tanto para 1991 quanto para 2000, tanto levando-se em conta a hipótese de contigüidade de primeira quanto de segunda ordem, contudo a autocorrelação de primeira ordem é maior que a de segunda. Isso que dizer que a influência dos vizinhos é maior quanto mais próximo este estiver do ponto de referência. A mesma observação é feita para o IPH-M, ou seja, o IPH-M de 1991 e de 2000 não se encontram aleatoriamente distribuídos, o que quer dizer que o índice de pobreza humana não depende somente de suas condições, mas também da situação dos vizinhos próximos.

Dessa forma, verifica-se a 'primeira lei da geografia' para a proporção dos pobres e para o IPH-M no Ceará, segundo a qual "todas as coisas são parecidas, mas coisas mais próximas se parecem mais que coisas mais distantes" (Tobler 1979 apud Chasco 2003:9).

TABELA 2. RESULTADO DO TESTE DE AUTOCORRELAÇÃO ESPACIAL MUNICIPAL, CEARÁ, 1991-2000

\begin{tabular}{ccccc}
\hline \multirow{2}{*}{ Ano } & \multicolumn{2}{c}{ I-Moran } & \multicolumn{2}{c}{ I-Moran } \\
\cline { 2 - 5 } & $\begin{array}{c}\text { Contigüidade de primeira ordem } \\
\text { Proporção de } \\
\text { pobres }\end{array}$ & IPH-M & $\begin{array}{c}\text { Pontigüidade de segunda ordem } \\
\text { pobres }\end{array}$ & IPH-M \\
\hline 1991 & 0,2205 & 0,2506 & 0,1410 & 0,1236 \\
2000 & 0,2734 & 0,2579 & 0,1360 & 0,1225 \\
\hline
\end{tabular}

FONTE: Elaboração do autor a partir dos dados do IPEADATA e a partir dos mapas georeferenciados disponíveis pelo IBGE. NOTA: Todas as estatísticas são significantes a $1 \%$.

Com a ajuda do indicador local de associação espacial (local indicator of spatial association - LISA), busca-se determinar as regiões no Ceará

10 Os seguintes municípios não apresentaram dados referentes a proporção dos pobres em 200o: Aceguá, Almirante Tamandararé do Sul, Arroio do Padre, Boa Vista do Cadeado, Boa Vista do Incra, Bozano, Canudos do Vale, Capão Bonito do Sul, Capão do Cipó, Coqueiro Baixo, Coronel Pilar, Cruzaltense, Forquetinha, Itati, Jacuzinho, Lagoa Bonita do Sul, Mato Queimado, Novo Xingu, Paulo Bento, Pedras Altas, Pinhal da Serra, Quatro Irmãos, Rolador, Santa Cecília do Sul, Santa Margarida do Sul, São José do Sul, São Pedro das Missões, Tio Hugo e Westfalia. 
nas quais são significativas as associações espaciais da pobreza municipal. Para a análise de contigüidade de primeira ordem (Figura 3), pode-se identificar que nos municípios sombreados existe algum tipo de relação espacial significativa entre os municípios e a proporção dos pobres. O mesmo foi feito para o IPH-M, na Figura 4. Dessa forma, há dois extremos: regiões com altos índices de pobreza (high-high no mapa) e áreas com baixos índices de pobreza (low-low no mapa).

Levando-se em conta a proporção de pobres, não há grande diferença da clusterização da pobreza no período analisado. As regiões com menor proporção de pobres mantiveram-se as mesmas: a primeira formada por Fortaleza, Aquiraz, Eusébio, Horizonte, Itaitinga, Maracanaú e Pacatuba, todos na messoregião metropolitana de Fortaleza; e a segunda formada por Alto Santo, São João do Jaguaribe, Limoeiro do Norte, Tabuleiro do Norte, Jaguaribara, Jaguaribe e Quixeré, todos da messoregião do Jaguaribe. Já as regiões com maior proporção de pobres modificam-se consideravelmente no período analisado. A clusterização da pobreza no noroeste cearense formado por Ipueiras, Catunda, Hidrolândia e Ipu deixa de apresentar indícios de clusterização em 2000 e se 'move' em direção aos municípios Marco, Senador Sá, Comocim, Chaval, Martinópole, Barroquinha e Granja, também no noroeste cearense.

Considerando-se o IPH-M a clusterização da região com menor índice de pobreza, ela é praticamente a mesma que a indicada pela proporção de pobres, contudo, há uma diferença significativa das regiões pobres levando em consideração o IPH-M e a proporção dos pobres, como pode ser visto a partir da análise visual dos mapas apresentados nas Figuras 3 e 4 .

A partir desse ponto, é possível verificar quais municípios passaram por um processo de difusão, já que em um lapso de tempo de praticamente uma década os municípios podem ter se modificado no plano cartesiano. De acordo com Díaz \& Sánchez (2004) a difusão espacial pode se dar a partir de dois efeitos: difusão espacial contagiosa e difusão espacial hierárquica. No primeiro caso, um município pode afetar um município vizinho, de modo que o último que era pobre (rico) passou a se classificar como rico (pobre). Já a difusão hierárquica ocorre sem nenhum tipo de contato espacial, ou seja, ocorre por imitação ou inovação. A difusão hierárquica não é o foco de análise desse artigo, já que estamos preocupados com a interação do espaço (ver Figura 5). 
FIGURA 3. ANÁLISE DE DEPENDÊNCIA ESPACIAL LOCAL (LISA) DA PROPORÇ̃̃O DE POBRES NOS MUNICÍPIOS CEARENSES, DE CONTIGÜIDADE DE PRIMEIRA ORDEM, 1991-2000
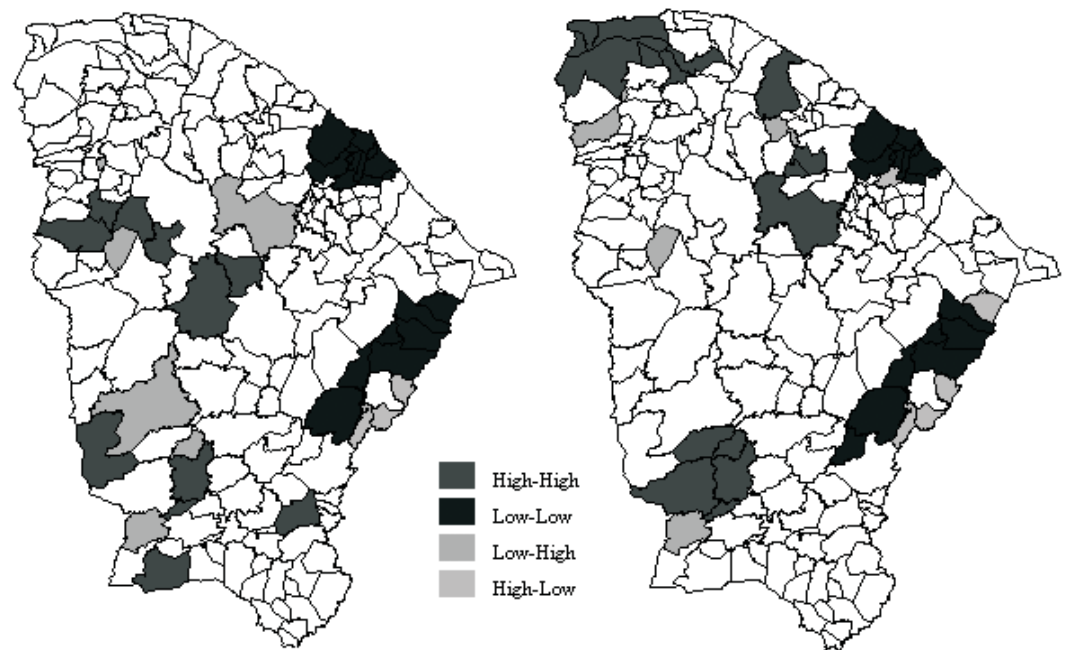

FONTE: Elaboração do autor a partir dos dados do Sistema Nacional de Indicadores Urbanos do Minisrio das Cidades e a prtir dos mapas geo-referenciados disponíveis pelo IBGE.

FIGURA 4. ANÁLISE DE DEPENDÊNCIA ESPACIAL LOCAL (LISA) DO IPHM NOS MUNICÍPIOS CEARENSES, DE CONTIGÜIDADE DE PRIMEIRA ORDEM, 1991-2000
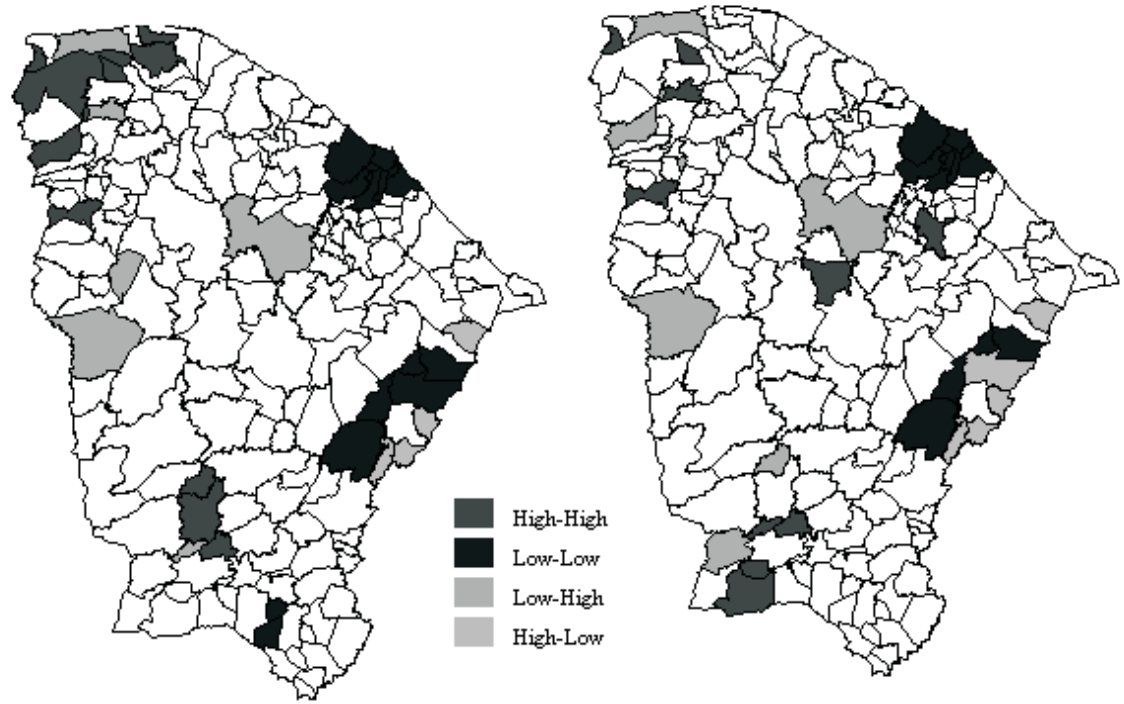

FONTE: Elaboração do autor a partir dos dados do Sistema Nacional de Indicadores Urbanos do Ministério das Cidades e a partir dos mapas geo-referenciados disponíveis pelo IBGE. 
A difusão contagiosa pode ocorrer por causa de re-alocação ou expansão (contração). No caso da re-alocação um município deixa de ser pobre (rico) sem afetar negativamente seus vizinhos. Já "la expansión o contracción, por su parte, implica que la situación de pobreza o riqueza cambia para el municipio local pero no para sus vecinos" (Perez 2005).

Fazendo a análise de difusão para o Ceará, chega-se aos resultados apresentados na Tabela 3. Observa-se que a proporção de pobres encontra-se em nove municípios que passaram por um processo de difusão de contágios por expansão enquanto que seis municípios passaram por um processo de difusão de contágios por contração. Já analisando o processo de difusão levando-se em conta o IPH-M, tem-se que sete municípios passaram por um processo de difusão de contágios por expansão, enquanto que seis municípios passaram por um processo de difusão de contágios por contração, ou seja, os setes municípios mencionados mudaram para a situação de riqueza local sem afetar seus vizinhos enquanto que os outros seis municípios referidos mudaram para a situação de pobreza local sem também afetar a seus vizinhos próximos.

\section{FIGURA 5. REPRESENTAÇÃO DOS TIPOS DE DIFUSÃO CONTAGIOSA}

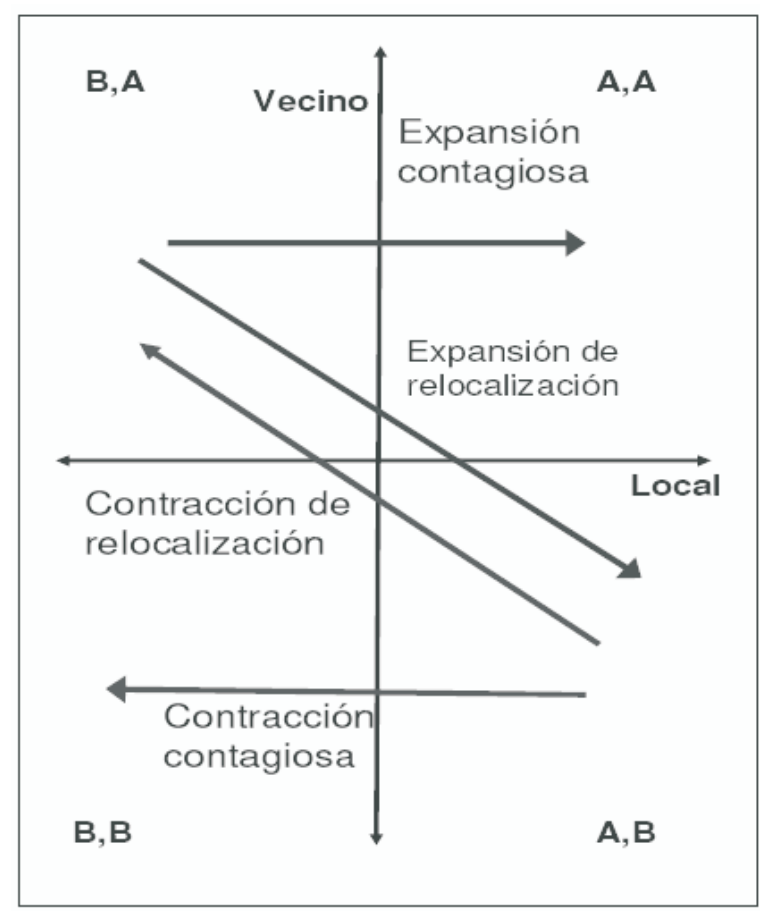

FONTE: Díaz \& Sánchez (2004:39). 
CHIARINI, T. Análise espacial da pobreza municipal no Ceará, 1991-200o

TABELA 3. MUNICÍPIOS CEARENCES QUE PASSARAM POR UM PROCESSO DE DIFUSÃO DE CONTÁGIOS, 1991-2000

\begin{tabular}{|c|c|c|c|c|}
\hline & & & \multicolumn{2}{|c|}{ Medidas de Pobreza } \\
\hline \multicolumn{3}{|c|}{ Processo de difusão por contágio } & Proporção de Pobres & IPH-M \\
\hline Cluster & 1991 a 2000 & $\begin{array}{l}\text { baixo-alto a } \\
\text { alto-alto }\end{array}$ & $\begin{array}{l}\text { Acaraú, Camocim, Candindé, } \\
\text { Catarina, Martinópolis, Massapê, } \\
\text { Moraújo, Pacujá e Santana do } \\
\text { Acaraú }\end{array}$ & $\begin{array}{c}\text { Acarapé, Antonina do Norte, } \\
\text { Aratuba, Independência, } \\
\text { Monsenhor Tabosa, Moraújo e } \\
\text { Potengi }\end{array}$ \\
\hline Cluster & 1991 a 2000 & $\begin{array}{l}\text { baixo-alto a } \\
\text { alto-baixo }\end{array}$ & Milagres e Quixelô & - \\
\hline Cluster & 1991 a 2000 & $\begin{array}{l}\text { alto-baixo a } \\
\text { baixo-alto }\end{array}$ & - & $\begin{array}{c}\text { Santa Quitéria e Santana do } \\
\text { Acaraú }\end{array}$ \\
\hline Cluster & 1991 a 2000 & $\begin{array}{l}\text { alto-baixo a } \\
\text { baixo-baixo }\end{array}$ & $\begin{array}{c}\text { Barreira, Fortim, Guaramiranga, } \\
\text { Jaguaretama, Nova Olinda e } \\
\text { Porteiras }\end{array}$ & $\begin{array}{c}\text { Caridade, Jati, Meruoca, } \\
\text { Milagres, Pindoretama, } \\
\text { Quixelô, }\end{array}$ \\
\hline
\end{tabular}

FONTE: Elaboração do autor a partir dos dados do Sistema Nacional de Indicadores Urbanos do Ministério das Cidades e a partir dos mapas geo-referenciados disponíveis pelo IBGE.

\section{Considerações finais}

Este trabalho utilizou-se da mapeação da pobreza e do instrumental econométrico espacial para fazer uma análise da pobreza nos municípios cearenses em 1991 e 2000 e ainda verificou se houve processo de difusão de contágios em alguns municípios do Ceará. Verificou-se a hipótese de que há dependência espacial da pobreza no Ceará tanto em 1991 quanto em 2000 com base na análise da estatística I-Moran, para ambos os indicadores de pobreza utilizados (proporção de pobres e IPH-M). Verificou-se ainda que existe clusterização tanto da miséria quanto da riqueza no Ceará (para ambos indicadores e em ambos períodos), fato amplamente reconhecido entre os cearenses. Verificou-se também, que mesmo havendo mantido a clusterização da pobreza, a mesma, tanto em termos de proporção de pobres quanto em IPH-M, vem diminuindo. Contudo, ela ainda continua em níveis elevados e políticas públicas devem ser aplicadas para aliviá-la.

A constatação de que a pobreza está espacialmente localizada no Ceará merece destaque. Dessa forma, estudos econométricos que tratam a incidência da pobreza cearense ou o IPH-M, como variáveis explicadas, devem levar em conta a influência do espaço sobre ela. Caso isso não seja feito corre-se o risco de encontrar conclusões equivocadas. Nesse novo contexto metodológico, as variáveis que podem explicar a pobreza cearense devem ser identificadas para que políticas públicas sejam aplicadas em locais realmente carentes. 


\section{Referências}

ANSELIN, Luc (1988). Spatial econometrics. Dordrecht (Netherlands): Kluwer Academic Publishers.

ANSELIN, Luc (1999a). "Spatial econometrics.” URL [On line]: http:// www.csiss.org/learning_resources/content/papers/baltchap.pdf. Acesso em: 10 de Janeiro de 2007.

ANSELIN, Luc (1999b). "The future of spatial analysis in the social sciences." Geographic information sciences 05 (02): 67-76.

ANSELIN, Luc (2000) "GIS, spatial econometris and social science research." Journal of Geographical Systems 02 (11): 11-15.

ANSELIN, L. \& REY, S. (1997). "Introduction to the special issue on spatial econometrics." International Regional Science Review 20 (01): 1-7.

ANSELIN, L. \& COHEN, J. \& COOK, D. \& GORR, W. \& TITA, G. (2000). "Spatial analysis of crime." In: DUFFEE, D. (ed). Measurement and analysis of crime and justice. Washington (DC): U.S. Department of Justice, pp. 213-62.

BORGES, Luciana (2004). "Mapa da pobreza urbana de São José dos Campos - SP 2000." Anais do XIV Encontro Nacional de Estudos Populacionais, ABEP, Caxambu, MG, 20-24 de setembro.

CÂMARA, G. \& MONTEIRO, A. \& RAMOS, F. \& SPOSATI, A. \& KOGA, D. (2004). "Mapping social exclusion/inclusion in developing countries: social dynamics of São Paulo in the 1990's." In JONELLE, D. \& GOODCHILD, M. (eds). Spatially-enabled social science: examples in best practice. New York: Oxford University Press, pp. 223-37.

CHASCO, Coro (2003) Econometría espacial aplicada a la predicciónextrapolación de datos microterritoriales. Madri (España): Consejería de Economía e Innovación Tecnológica.

CUNHA, Maria Beatriz A. (2006). Mapas de pobreza: avaliação da metodologia e de sua aplicação para retratar a pobreza no Rio Grande do Norte. Rio de Janeiro: dissertação de mestrado, Escola Nacional de Ciências Estatísticas (ENCE).

DAVIS, Benjamin (2002). "Is it possible to avoid a lemon? Reflections on choosing a poverty mapping method." Food Policy and Applied Nutrition Program, discussion paper n. 08.

DAVIS, Benjamin (2003). "Choosing a method for poverty mapping." Roma: FAO, UN.

DEICHMANN, Uwe (1999). "Geographic aspects of inequality and poverty." URL [On line]: http://www.worldbank.org/poverty/inequal/index.htm. Acesso em: 21 de dezembro de 2006.

DÍAZ, Ana Maria \& SÁNCHEZ, Fabio (2004). "Geografia de los cultivos ilícitos y conflicto armado en Colombia." Documento DECE, n. 2004-18, Centro de Estúdios Sobre Desarollo Económico de la Faculdad de Economía de la Universidad de los Andes.

GAUCI, Adrian (2005). "Spatial maps: targeting and mapping poverty." 
United Nations Economic Commission for Africa. Working paper.

GENOVEZ, Patrícia C. (2002). Território e desigualdade: análise espacial intra-urbana no estudo da dinâmica de exclusão/inclusão social no espaço urbano de São José dos Campos. São José dos Campos: dissertação de mestrado, Instituto Nacional de Pesquisas Espaciais (INPE).

HAGENAARS, Aldi (1987) “A class of poverty indices." International economic review 28 (3): 583-607.

HAGENAARS, Aldi \& DE VOS, Klaas (1988). "The definition and measurement of poverty." The journal of human resources 23 (2): 211221.

HENNINGER, Norbert (1998). "Mapping and geographic analysis of human welfare and poverty: review and assessment." World Resources Institute, Washington, D.C.

HENNINGER, Norbert \& SNEL, Mathilde (2002). "Where are the poor? Experiences with the development and use of poverty maps." World Resources Institute, Washington, D.C.

HENTSCHEL, J. \& LANJOUW, J. \& LANJOUW, P. \& POGGI, J. (1998). "Combining census and data to study spatial dimensions of poverty." Policy Research Working Paper 1928. The World Bank, Washington, USA.

IPEA (2007). IPEAdata. Rio de Janeiro: Ministério do Planejamento, Orçamento e Gestão. URL [On line]: http://www.ipeadata.gov.br/. Acesso em: 13 de agosto de 2007.

LESAGE, James P. (1998). “Spatial econometrics.” URL [On line]: http:// www.spatial-econometrics.com/html/wbook.pdf. Acesso em: 10 de janeiro de 2007.

LIBERATO, Vânia C. (2004). "Linhas de indigência e pobreza para Belo Horizonte, RMBH e colar metropolitano." In: LEMOS, M. \& DINIZ, C. \& CARVALHO, J. M. \& SANTOS, F. (coord.). Projeto Belo Horizonte no século $X X I$. Belo Horizonte: CEDEPLAR. URL [On line]: http:// www.cedeplar.ufmg.br/pesquisas/projeto_pbh_sec._xxi.php. Acesso em: 27 de janeiro de 2007.

McKINLEY, Terry (1997). "Beyond the line: implementing complementary methods of measurement." In: DESSALLIEN, Renata (ed.). Technical support document, poverty reduction, module 3 - poverty measurement: behind and beyond the poverty line. UNDP.

OLIVEIRA, Cristiano A. (2006). "Uma Análise Espacial dos Impactos do Crescimento Econômico e da Concentração de Renda na Pobreza dos Municípios Nordestinos na Década de Noventa." Anais do XI Encontro regional de economia, ANPEC Nordeste, Fortaleza.

PÉREZ, Gerson Jr. (2005). "Dimensión espacial de la pobreza en Colombia.” Banco de la República, Documentos de trabajo sobre economía regional, n. 54 .

PETRUCCI, A. \& SALVATI, N. \& SEGHIERI, C. (2003). "The application of a spatial regression model to the analysis and mapping of poverty." Environment and Natural Resources Series 07, Roma: FAO, UNI. 
PORTO ALEGRE (2004). "Mapa da inclusão e exclusão social de Porto Alegre." Prefeitura de Porto Alegre, Secretaria do Planejamento Municipal. URL [On line]: http://lproweb.procempa.com.br/pmpa/prefpoa/observatorio/ usu_doc/MapaIncExcPOA.pdf. Acesso em: 27 de janeiro de 2007.

RAVALLION, Martin (1992). "Poverty comparisons: a guide to concepts and methods." The World Bank, Washington, LSMS Working Paper, n. 88.

ROMERO, Julio A. R. (2006). "Análise especial da pobreza municipal do estado de Minas Gerais - 1991-2000." ANAIS do XIV Encontro Nacional de Estudos Populacionais, ABEP, Caxambu.

UNDP (1996). Human Development Report: economic growth and human development. Nova Iorque: UNDP.

UNDP (1997). Human Development Report: human development to eradicate poverty. Nova Iorque: UNDP.

UNDP (2004). Human Development Report: cultural liberty in today's diverse world. Nova Iorque: UNDP.

VAYÁ, Esther V. \& MORENO, Rosina (2000). "La utilidad de la econometría espacial en el ámbito de la ciencia regional." Fundación de Estudios de Economia Aplicada, Madrid, España. Documento de Trabajo 2000-13.

Submissão: 15 de maio de 2007 Primeira resposta: 13 de julho de 2007 Aceite: 14 de agosto de 2007 
CHIARINI, T. Análise espacial da pobreza municipal no Ceará, 1991-200o

\section{ANEXO - Diagrama de dispersão I-Moran.}

FIGURA 1A. SCATTERPLOT DE MORAN PARA VERIFICAR A AUTOCORRELAÇÃO ESPACIAL DA PROPORÇÃO DE POBRES DOS MUNICÍPIOS CEARENSES, EM 1991-2000, COM CONTIGÜIDADE DE PRIMEIRA E SEGUNDA ORDEM, RESPECTIVAMENTE.
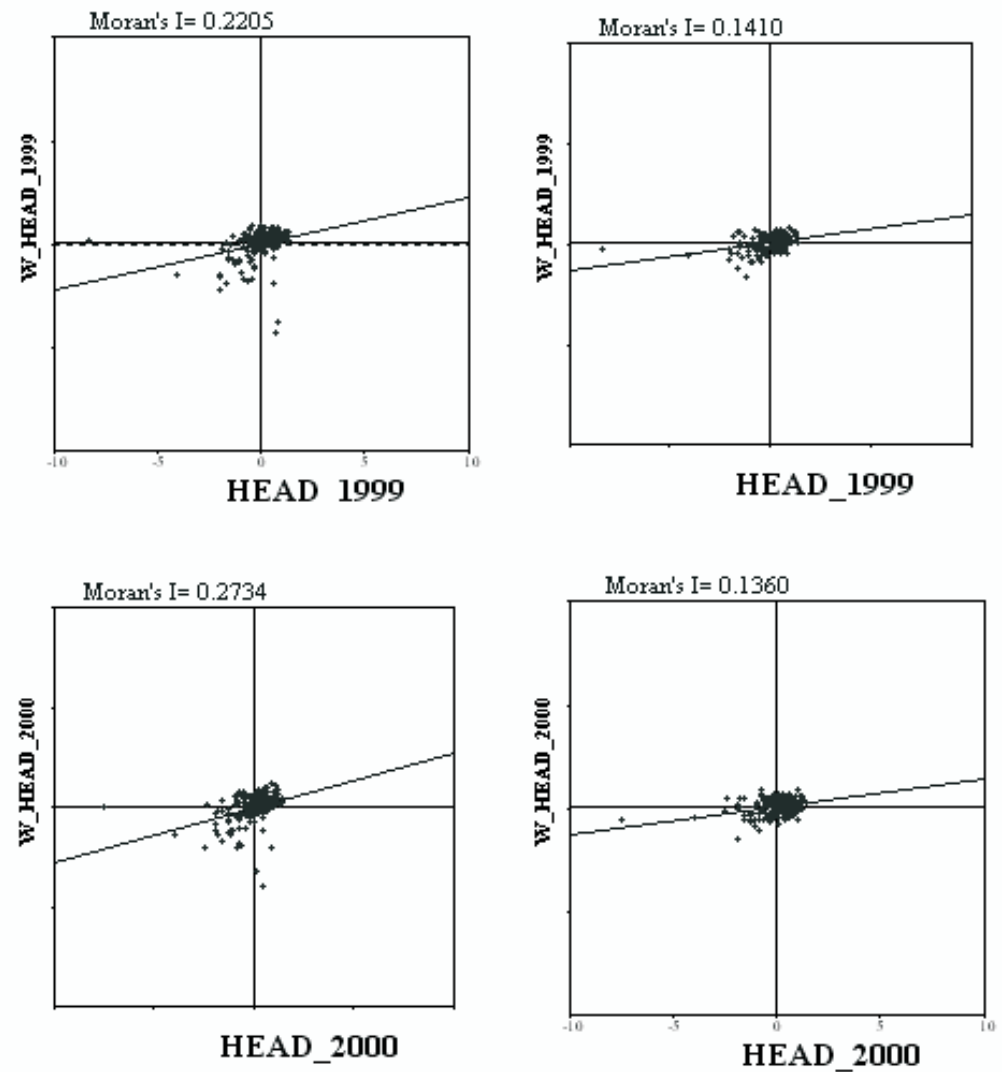

FONTE: Construção do autor a partir dos dados do IPEADATA edos mapas georeferenciados disponíveis pelo IBGE. 
FIGURA 2A. SCATTERPLOT DE MORAN PARA VERIFICAR A AUTOCORRELAÇÃO ESPACIAL DO IPH-M DOS MUNICÍPIOS CEARENSES, EM 1991-2000, COM CONTIGÜIDADE DE PRIMEIRA E SEGUNDA ORDEM, RESPECTIVAMENTE.
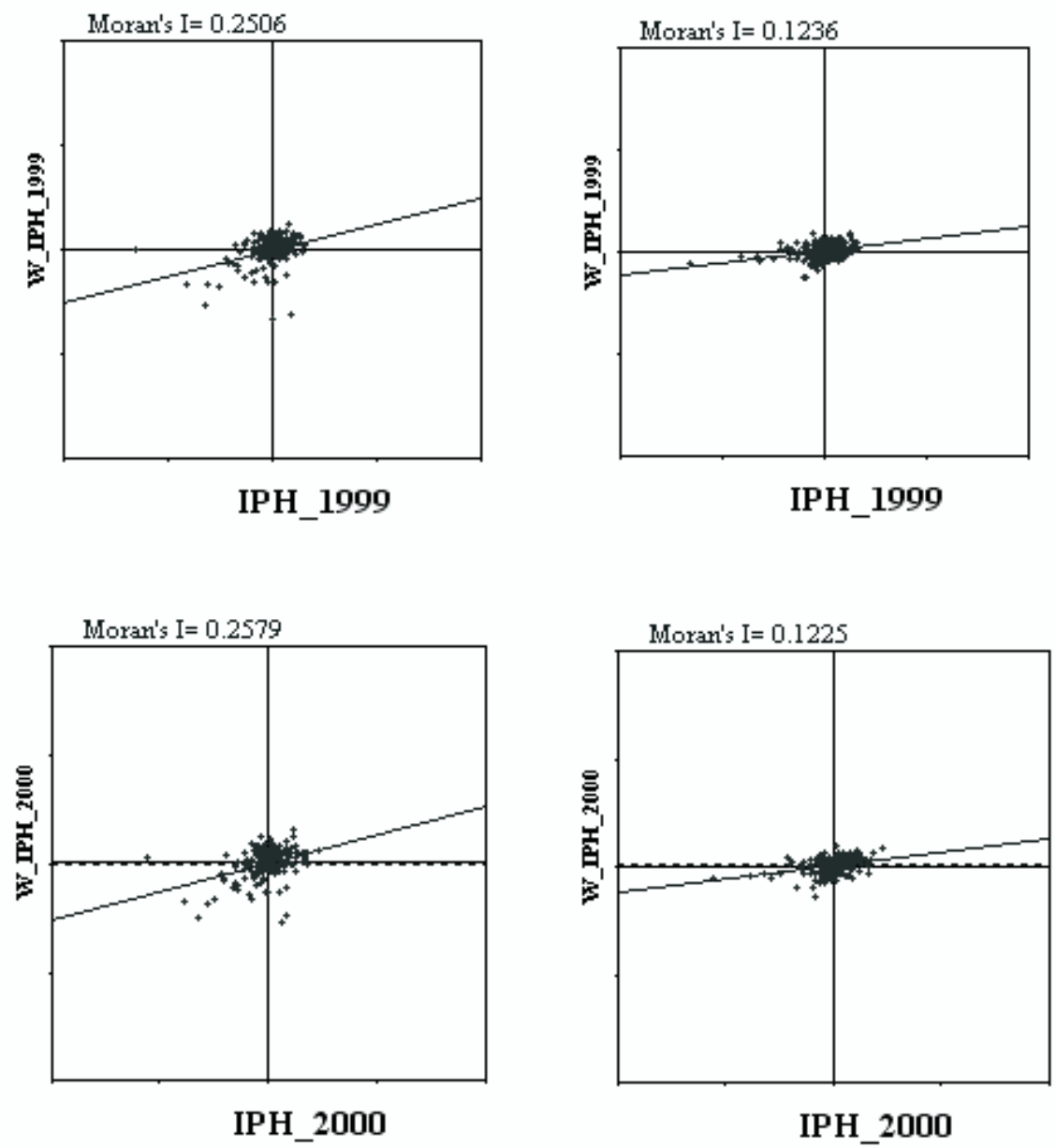

FONTE: Construção do autor a partir dos dados do IPEADATA e dos mapas georeferenciados disponíveis pelo IBGE. 